ARTICLE

DOI: $10.1038 / s 41467-017-00462-2$

\title{
Misalignment with the external light environment drives metabolic and cardiac dysfunction
}

\author{
Alexander C. West ${ }^{1}$, Laura Smith ${ }^{1}$, David W. Ray (1) ${ }^{1}$, Andrew S.I. Loudon', Timothy M. Brown ${ }^{1}$ \\ $\&$ David A. Bechtold (10 ${ }^{1}$
}

Most organisms use internal biological clocks to match behavioural and physiological processes to specific phases of the day-night cycle. Central to this is the synchronisation of internal processes across multiple organ systems. Environmental desynchrony (e.g. shift work) profoundly impacts human health, increasing cardiovascular disease and diabetes risk, yet the underlying mechanisms remain unclear. Here, we characterise the impact of desynchrony between the internal clock and the external light-dark (LD) cycle on mammalian physiology. We reveal that even under stable LD environments, phase misalignment has a profound effect, with decreased metabolic efficiency and disrupted cardiac function including prolonged QT interval duration. Importantly, physiological dysfunction is not driven by disrupted core clock function, nor by an internal desynchrony between organs, but rather the altered phase relationship between the internal clockwork and the external environment. We suggest phase misalignment as a major driver of pathologies associated with shift work, chronotype and social jetlag.

\footnotetext{
${ }^{1}$ Division of Diabetes, Endocrinology and Gastroenterology, School of Medicine, Faculty of Biology, Medicine and Health, University of Manchester, Manchester Academic Health Science Centre, Manchester M13 9PL, UK. Correspondence and requests for materials should be addressed to D.A.B. (email: david.bechtold@manchester.ac.uk)
} 
T he periodic succession of night and day influences nearly all forms of life on earth. As a result, organisms have evolved internal circadian clocks capable of keeping near precise $24 \mathrm{~h}$ time. By tracking time internally, organisms adapt their biology to match cyclical fluctuations in the environment (e.g. light, food availability, predation) and thus respond optimally. In mammals, the circadian system consists of a network of tissue clocks housed across the body and coordinated by a central pacemaker located within the suprachiasmatic nucleus (SCN) of the anterior hypothalamus. Within all of these sites, the molecular clock machinery drives rhythmic transcriptional and metabolic pathways in a tissue-specific manner, a process critical to proper tissue function ${ }^{1-3}$. Altered circadian function, be it through genetic variation, lifestyle factors (e.g. chronic shift work, sleep restriction, nocturnal light exposure) or experimental perturbation (e.g. forced desynchrony) are linked to a wide range of pathogenic states from metabolic disease to cancer ${ }^{4-9}$. A further, more prevalent circadian insult occurs when the phase of our internal timing (chronotype) does not match with patterns of societal-driven activity (commonly referred to as social jet-lag). Indeed, large population and targeted cohort studies are now linking chronotype with a variety of health, psychiatric and life history variables ${ }^{10-13}$. Given that modern life has disturbed the natural temporal structure of our environment, it is critical that we understand the mechanisms which link clock desynchrony to pathophysiological outcomes.
In mammals, circadian timekeeping is centred on the feedback coupling of the transcriptional activators CLOCK and BMAL1, and repressors PERIOD, CRYPTOCHROME and REVERB. Much of our understanding of clock function has been defined through genetic ablation of these and additional constituent factors ${ }^{14}, 15$. Animal studies demonstrate that genetic disruption of individual core clock genes can have a severe impact on heath, ranging from disturbances in metabolism and inflammatory response, to altered bone formation and neurodegeneration ${ }^{14}, 16,17$. However, due to pleotropic and/or developmental activity of the targeted clock genes, it is rarely possible to isolate the influence of clock timing per se from that of the ablated factor ${ }^{18,19}$. Moreover, in the context of human health, misalignment with the environment is overwhelmingly the principal source of circadian disruption, rather than the relatively minor contributions of genetic disruption in clock gene function.

Despite human circadian desynchrony being widely acknowledged as deleterious to health, the reasons for increased risk remain unclear, and the pathways to disease undefined. Here, we recapitulate in mice the circadian misalignment that occurs during shift work and in human subjects with extremechronotype $e^{20-22}$. We show that long-term housing of mice under light-dark (LD) cycles that do not match a normal 24 $\mathrm{h}$ cycle leads to pronounced physiological disturbance, including altered metabolic efficiency and substrate utilisation, and a profound depression of cardiac function, including significant prolongation of PR and QT intervals. Our study
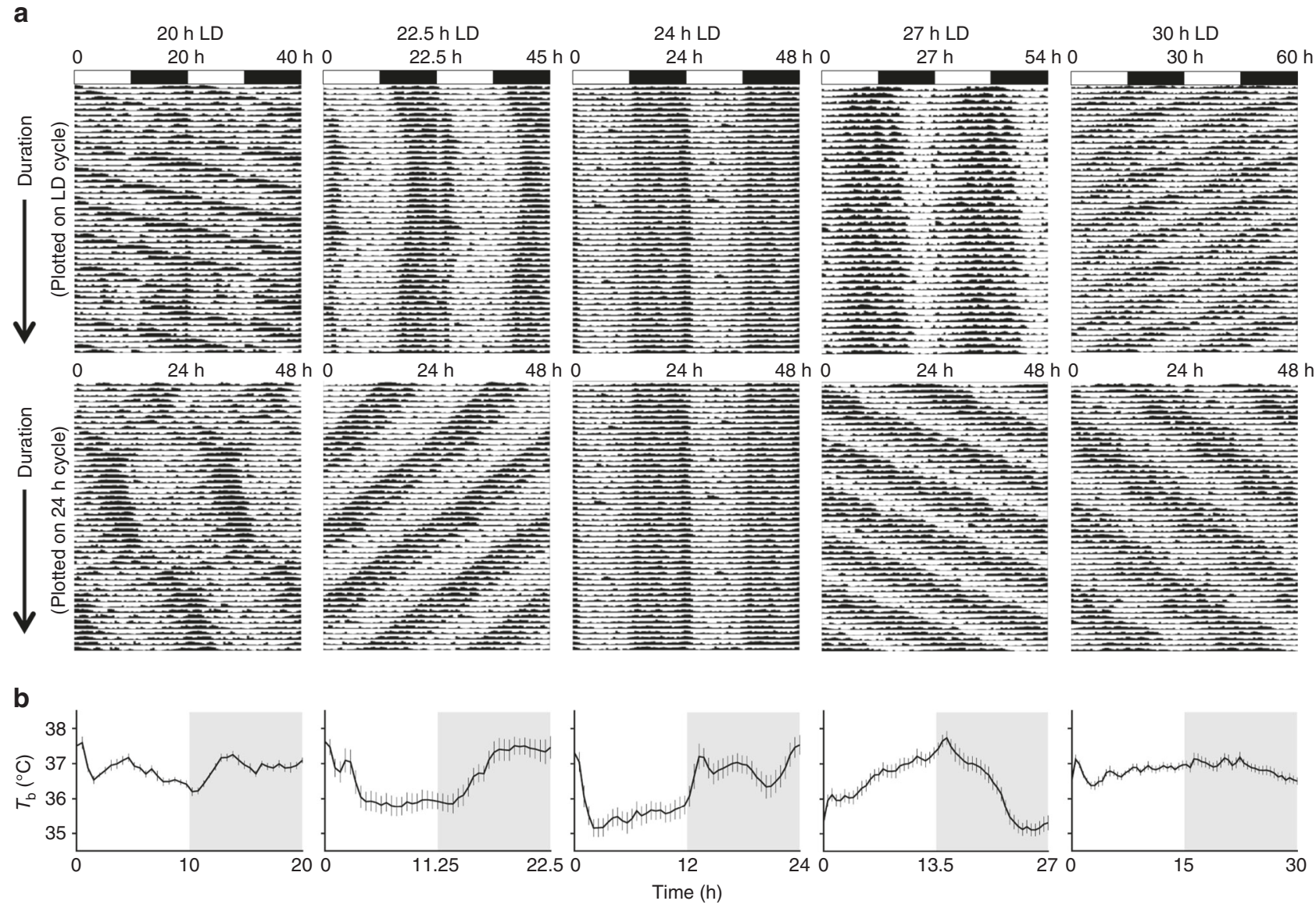

Fig. 1 Long-term housing under non-24h LD cycles drives phase desynchrony between physiological rhythms and the LD cycle. a Representative body temperature $\left(T_{\mathrm{b}}\right)$ rhythms from mice maintained on 20, 22.5, 24, 27 and $30 \mathrm{~h} \mathrm{LD}$ cycles. Top panels are double plotted relative to LD cycle time; bottom panels are plotted on $24 \mathrm{~h}$ frequency. $\mathbf{b}$ Group $T_{\mathrm{b}}$ profiles across the LD cycle (derived from 10 consecutive cycles, after $40 \mathrm{~d}$ LD exposure) highlight the delayed $(22.5 \mathrm{~h})$ and advanced $(27 \mathrm{~h}$ ) phase of entrainment in non-24 h LD housed mice. Data in $\mathbf{b}$ reflect mean \pm SEM, $n=7 /$ group for 20 and $30 \mathrm{~h}$ LD; $n=10 /$ group for $22.5,24$ and $27 \mathrm{~h} \mathrm{LD}$ 
a

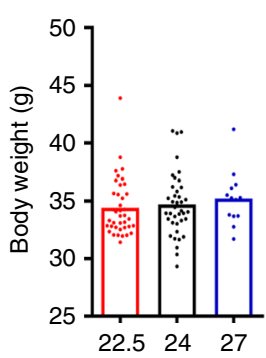

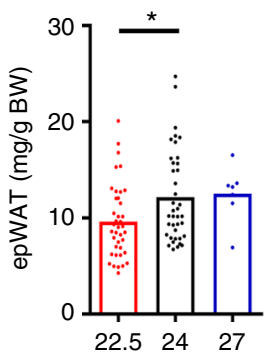

b
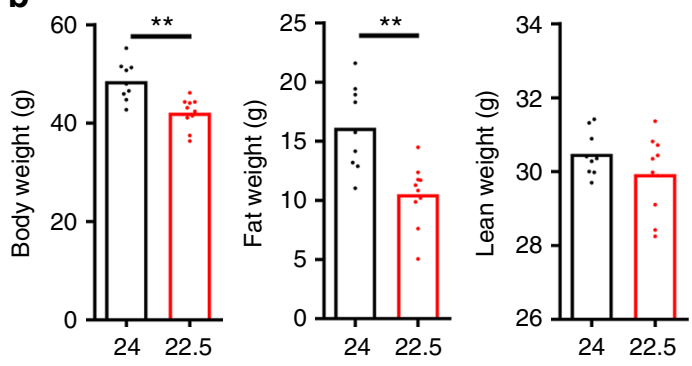

c
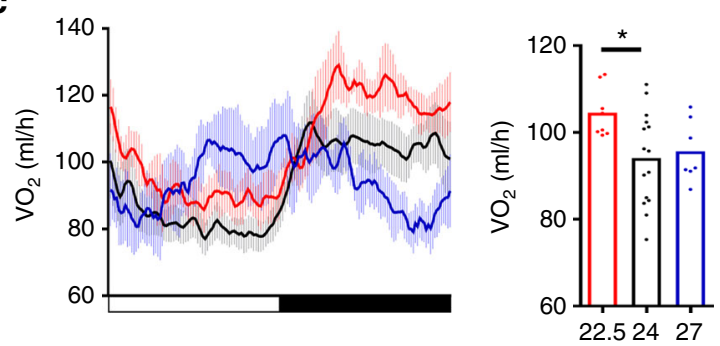

d
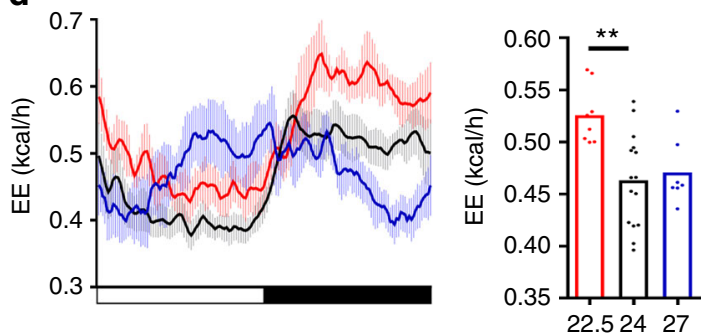

e
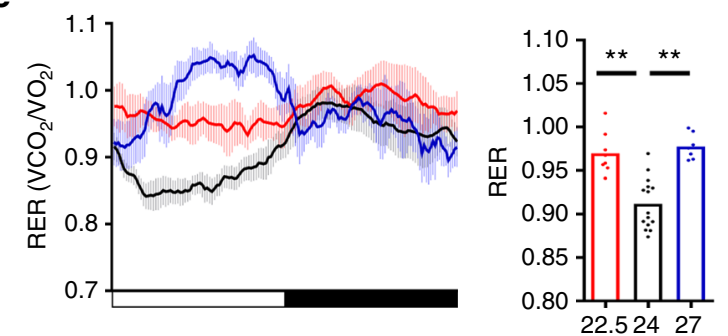

f
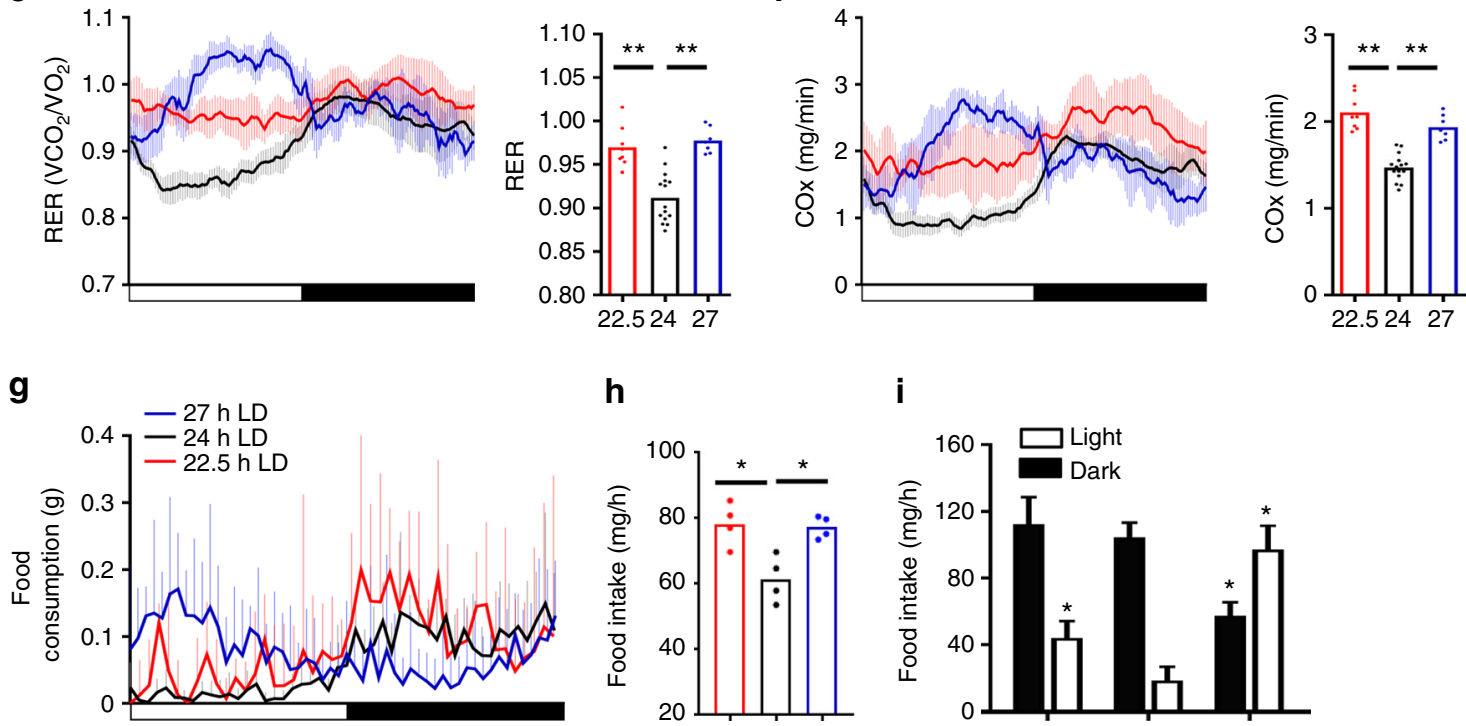

h

i
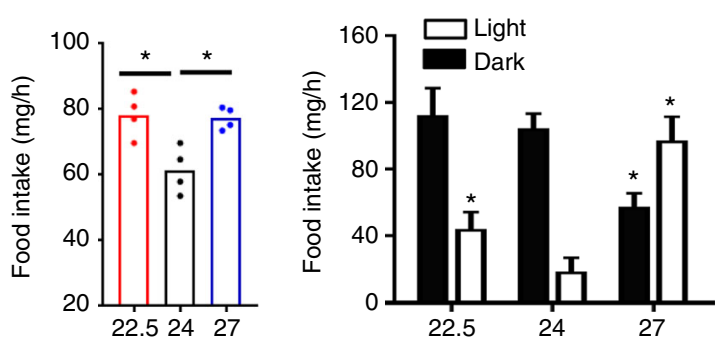

Fig. 2 Phase misalignment drives reduced energy efficiency and metabolic disturbance. a Body weight and epididymal adipose tissue mass (epWAT) of mice maintained in $22.5,24$ or $27 \mathrm{~h} \mathrm{LD}$ conditions for 17 week. epWAT was significantly lower in $22.5 \mathrm{~h} \mathrm{LD}$ mice compared with age-matched $24 \mathrm{~h}$ controls ( $n>7$ /group). b By 1 year of LD exposure, both body weight and whole-body fat mass were significantly lower in $22.5 \mathrm{~h}$ LD housed mice compared with matched $24 \mathrm{~h} \mathrm{LD} \mathrm{mice} \mathrm{(} n=9-10 /$ group). c-f Diurnal rhythms and mean values for oxygen consumption $\left(\mathrm{VO}_{2} ; \mathbf{c}\right)$, energy expenditure (EE; d), respiratory exchange rate (RER; e) and carbohydrate oxidation (COx; f) for mice maintained under 22.5, 24 and 27 h LD cycles ( $n=7-16 /$ group). A significant increase in RER and COx was evident in non-24 h LD housed mice. g-i Diurnal profiles of food intake ( $\mathbf{g}$ ) and mean intake/h (h) reveal significantly increased food consumption in mice maintained under either 22.5 or $27 \mathrm{~h}$ LD cycles compared with $24 \mathrm{~h}$ LD control. Mice housed under non-24 $\mathrm{h}$ LD cycles also consumed more food during the light phase of the cycle (i). Diurnal profiles $\mathbf{c}-\mathbf{g}$ reflect mean $\pm 95 \% \mathbf{C l} . \mathbf{a}, \mathbf{c}-\mathbf{h} * p<0.05, * * p<0.01$ one-way ANOVA with Dunnett's post hoc test (vs. $24 \mathrm{~h}$ control group); $\mathbf{b}^{* *} p<0.01$ Students $t$-test; $\mathbf{i}^{*} p<0.05$ vs. $24 \mathrm{~h}$ LD two-way ANOVA with Tukey's post hoc test

highlights the importance of phase alignment of the circadian clock to the environment and implicates disrupted entrainment, common place in the modern world, as being a major driver of pathology.

\section{Results}

Entrainment to non-24 h light cycles leads to reduced energy efficiency. To establish an altered phase relationship between the environmental LD cycle and the internal circadian clock, male C57Bl/6J mice were placed under $20 \mathrm{~h}, 22.5 \mathrm{~h}, 24 \mathrm{~h}, 27 \mathrm{~h}$ and
$30 \mathrm{~h}$ symmetrical LD cycles for 17 week (from 8 week of age, $n>8$ /group). Mice maintained under $20 \mathrm{~h}$ or $30 \mathrm{~h}$ LD cycles were unable to entrain, and exhibited a free-running rhythm with a high degree of phase dispersion between individual animals (Fig. 1; Supplementary Fig. 1). In contrast, mice maintained in $22.5 \mathrm{~h}, 24 \mathrm{~h}$ and $27 \mathrm{~h}$ conditions achieved stable entrainment in locomotor activity and core body temperature $\left(T_{\mathrm{b}}\right)$. However, in comparison to $24 \mathrm{~h} \mathrm{LD}$ housed mice, the phase of behavioural or physiological rhythms (as defined by the acrophase of $T_{\mathrm{b}}$ and activity) were respectively delayed or advanced relative to the onset of night under $22.5 \mathrm{~h}$ and $27 \mathrm{~h}$ 
conditions (Fig. 1a, b; Supplementary Fig. 1). Thus, the high degree of phase coordination among individual mice and the long-term stability of their phase alignment relative to the LD cycle make the $22.5 \mathrm{~h}$ and $27 \mathrm{~h} \mathrm{LD}$ cycle conditions ideal to test the physiological consequence of environmental desynchrony.

Energy metabolism is tightly coupled to the circadian clock $^{23-25}$; therefore, the impact of $22.5 \mathrm{~h}$ and $27 \mathrm{~h} \mathrm{LD}$ environment on body weight, adiposity, feeding behaviour and energy expenditure was examined. In comparison to matched 24 $h$ LD housed mice, a significant reduction in adiposity was observed in $22.5 \mathrm{~h}$ LD conditions (Fig. 2a). No significant differences were observed in comparable analyses of mice housed under $27 \mathrm{~h} \mathrm{LD}$ conditions. Reduced fat mass in $22.5 \mathrm{~h}$ LD mice was exacerbated by 12 months of $22.5 \mathrm{~h}$ LD exposure, by which time a significantly lower body weight was also evident (Fig. 2b). Consistent with reduced adiposity, $22.5 \mathrm{~h}$ LD housed

a

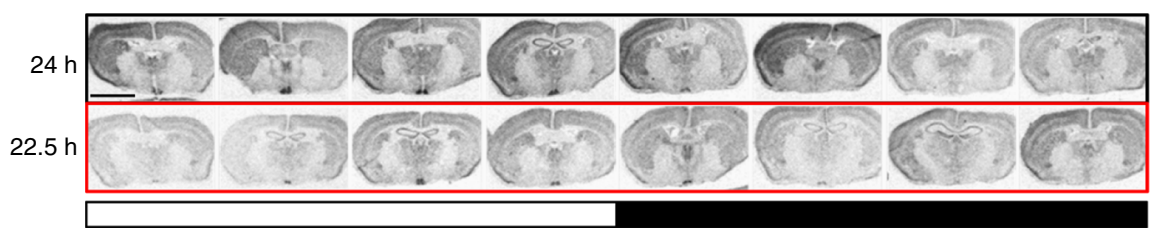

b

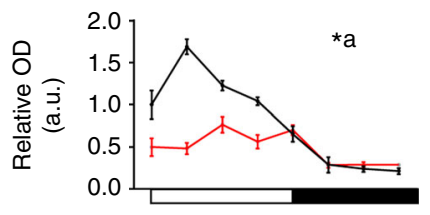

C

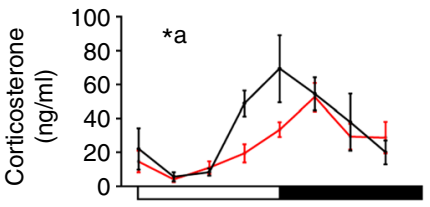

d

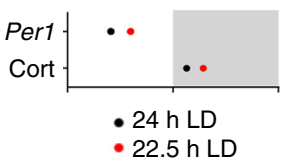

e
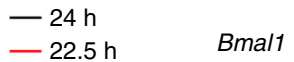

Per2

Cry1

Nr1D1

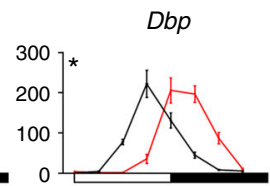

Lung

Liver
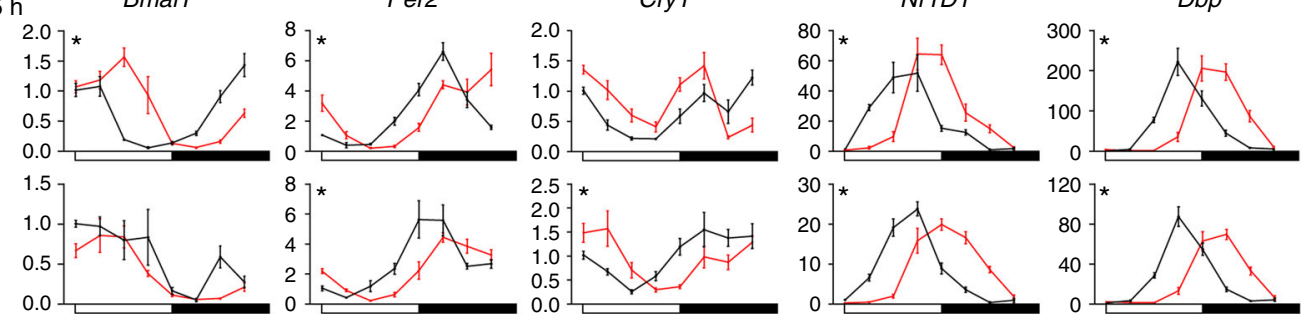

Adrenal
gland

Heart

Kidney
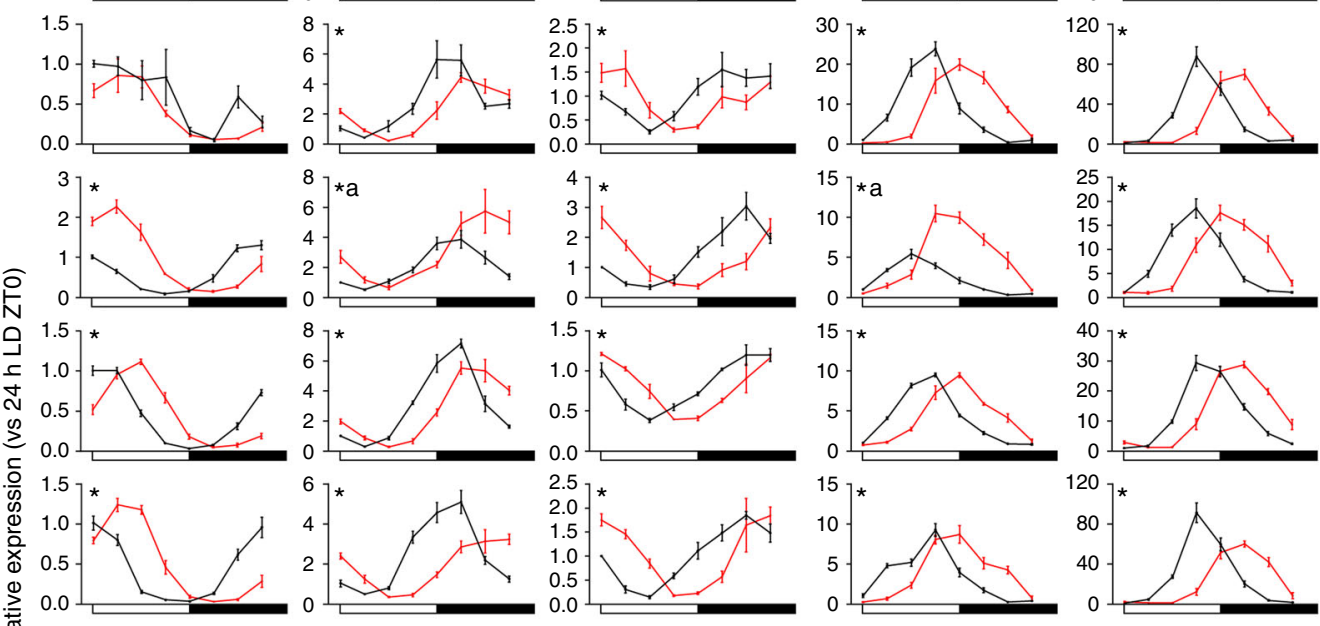

epWAT
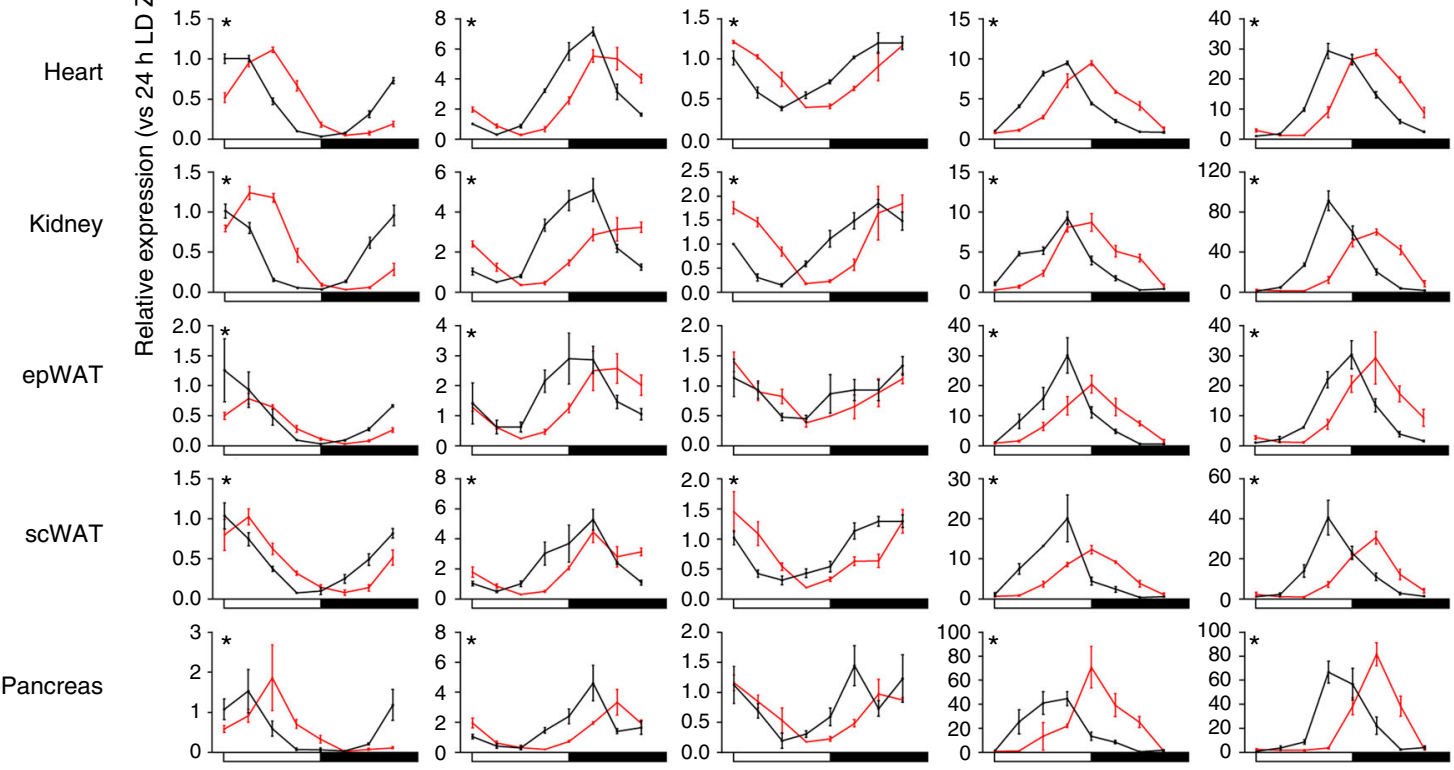

$\mathbf{f}$
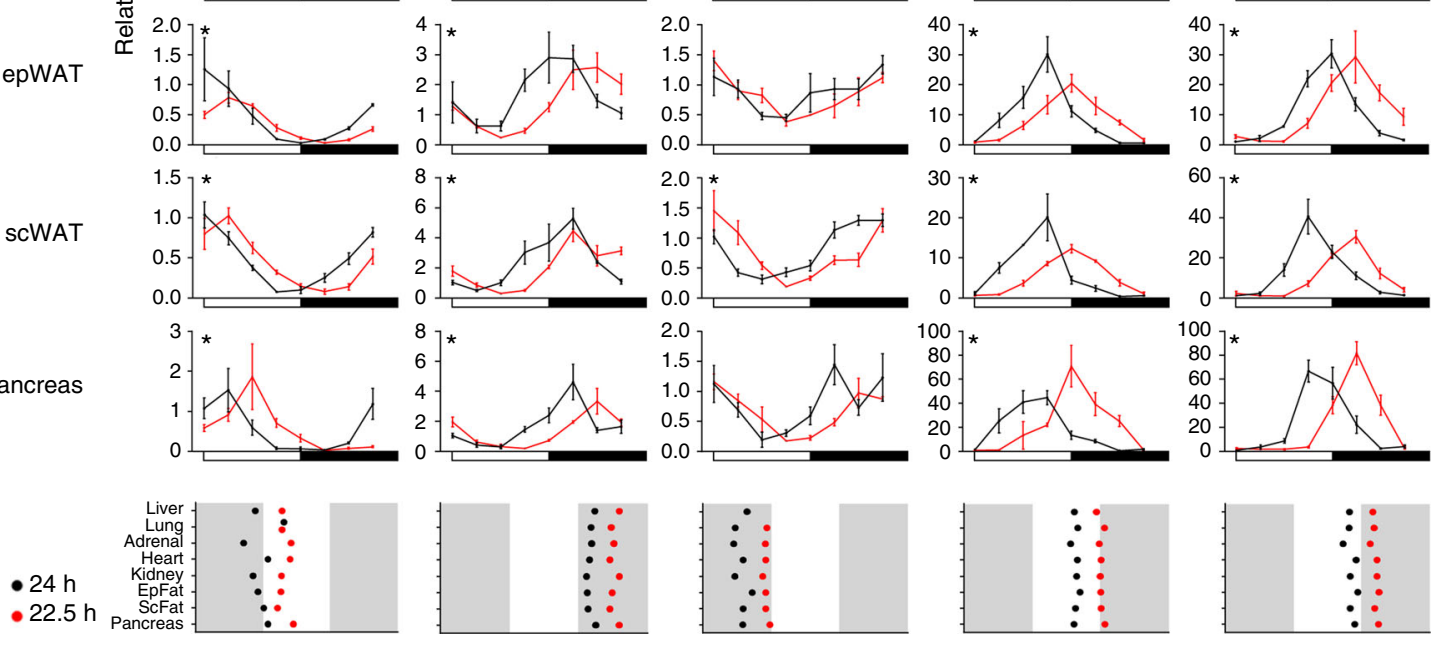
mice exhibited higher rates of both oxygen consumption $\left(\mathrm{VO}_{2}\right.$; Fig. 2c) and energy expenditure (EE; Fig. 2d). Interestingly, both the $22.5 \mathrm{~h}$ and $27 \mathrm{~h} \mathrm{LD}$ conditions caused a significant increase in respiratory exchange rate (RER) and carbohydrate oxidation $(\mathrm{COx})$ rate (Fig. 2e, f), indicating an increased reliance on carbohydrate substrates for energy generation in these mice. To assess feeding behaviour, mice were temporarily housed in automated feeding cages, and food intake monitored continuously for 5 days. In line with the increase in RER and COx, mice housed under non-24 h LD conditions exhibited a significant increase in food intake when compared with matched $24 \mathrm{~h} \mathrm{LD}$ controls (Fig. $2 \mathrm{~g}, \mathrm{~h}$ ), which was due in large part to an increased intake during the light phase of the cycle (Fig. 2i). Together these findings show that entrainment to non- $24 \mathrm{~h} \mathrm{LD}$ cycles has a pronounced effect on energy efficiency (higher food intake without increased body weight) and substrate utilisation.

Entrainment to non-24h LD cycles does not disrupt clock function. Despite the clear phase misalignment relative to the light cycle, mice entrained to non-resonant LD cycles exhibited a robust amplitude and consistent phase across different physiological parameters (Supplementary Fig. 1), suggesting that internal circadian timing is maintained in the animals. Therefore, we next sought to identify how central and peripheral oscillators respond to non- $24 \mathrm{~h} \mathrm{LD}$ cycles, with a focus on the $22.5 \mathrm{~h} \mathrm{LD}$ condition. Quantification of Perl in the master SCN clock revealed a peak in expression during the early light phase within the $24 \mathrm{~h} \mathrm{LD}$ housed mice (Fig. 3a, b). In line with the altered timing of activity and $T_{\mathrm{b}}$ in $22.5 \mathrm{~h} \mathrm{LD}$ housed mice, the rhythm in Per1 expression in the SCN of these mice was significantly delayed in phase and dampened in amplitude relative to the $24 \mathrm{~h}$ LD group (Fig. 3a, b). This was reflected in circulating corticosterone, an important synchronising agent for peripheral tissue clocks whose secretion is strongly influenced by the $\mathrm{SCN}^{26}$. Corticosterone rhythms were significantly delayed in phase in mice entrained to $22.5 \mathrm{~h}$ LD cycles, when compared with $24 \mathrm{~h}$ LD housed animals (Fig. 3c, d). Rhythms in both SCN gene expression and corticosterone were significantly dampened in the $22.5 \mathrm{~h} \mathrm{LD}$ housed mice, suggesting that the phase misalignment with the external LD cycle weakens the central oscillator.

Contrary to expectation, transcript profiles in peripheral tissues remained strongly rhythmic in both $24 \mathrm{~h}$ and $22.5 \mathrm{~h}$ LD housed groups (Fig. 3e). Strikingly, acrophase analyses revealed a consistent phase-delay relative to the LD cycle across all of the peripheral tissues examined (Fig. 3f), similar to that observed in the SCN. There was also a remarkable preservation of the phase relationship of clock components, both within and between tissue sets. A few exceptions were evident (e.g. Bmall expression in the lung), but these were limited to single gene profiles in individual tissues. Thus, despite chronic entrainment to a short-running LD cycle, the circadian system remains intact and faithfully retains its amplitude and internal synchrony.

Many cellular processes including metabolism ${ }^{27}$, cell cycle $^{28}$, oxidative stress $^{29}$, inflammation ${ }^{30}$ and epigenetic modification ${ }^{31}$ are subject todirect transcriptional control bycomponents of the clock. Therefore, we next profiled regulatory factors and pathways known to be under circadian control (Fig. 4; Supplementary Fig. 2). The expression profile of manyfactors (e.g. wee1) matched that of the core clock, remaining robustly rhythmic but delayed in phase under $22.5 \mathrm{~h}$ LD conditions. However, many other factors (e.g. $n r f 2$, pparg, ezh2, RelA) exhibited significant transcriptional dysregulation,with alterations in amplitude and/or mesor. Dysregulation of affectedpathways (e.g. antioxident response, histone/DNA methylation) was highly tissue specific, demonstrating the tissue selective impact of phase misalignment (Supplementary Fig. 2). Thus,despite maintenance of robust core circadian clock function in non-resonant mice, disruption of timing was evident in a number of key clock-output pathways.

Exposure to non-resonant LD cycles impairs cardiac function.

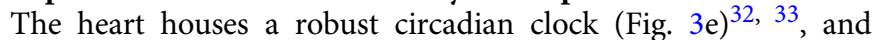
cardiovascular health is adversely affected by shift work and forced desynchrony protocols ${ }^{9}, 34$. We therefore examined the impact of $22.5 \mathrm{~h}$ and $27 \mathrm{~h} \mathrm{LD}$ cycles on heart rate (HR) and electrocardiogram (ECG) parameters. Mice exhibited a robust diurnal rhythm in HR under $24 \mathrm{~h} \mathrm{LD}$; however both $22.5 \mathrm{~h}$ and $27 \mathrm{~h} \mathrm{LD}$ cycles disrupted the HR rhythms, and significantly slowed HR across the LD cycle (Fig. 5a; Supplementary Fig. 1d-f). The reduction in HR was not related to changes in locomotor activity (Fig. 5b; analyses limited to periods of $>20 \mathrm{~min}$ of inactivity before HR measure).Critically, longitudinal analyses of ECG recordings in wild-type C57B6J mice revealed that non-resonant LD conditions not only increased inter-beat (RR) interval (Fig. 5d), but also slowed cardiac conduction parameters, with significantly lengthening of PR, QT and RR-adjusted QT (QTc) intervals evident in mice housed under either 22.5 or $27 \mathrm{~h}$ LD cycles (Fig. 5e-h).

Prolonged inter-beat intervals can result from changes in repolarising potassium channels ${ }^{35}$. However, we did not observe aberrant ion channel expression in the $22.5 \mathrm{~h} \mathrm{LD}$ mice (Supplementary Fig. 2). In our studies, HR slowing occurred rapidly upon transition from $24 \mathrm{~h}$ to non-resonant $\mathrm{LD}$ cycles, and was evident in mice switched from $24 \mathrm{~h} \mathrm{LD}$ to constant light (Supplementary Fig. 3). A similar LD cycle-dependent slowing of HR was observed in the accelerated period CK1 $\varepsilon^{\text {tau }}$ mutant mice (free-running period of $20 \mathrm{~h}$ ), when housed under $22.5 \mathrm{~h} \mathrm{LD}$ cycles (Supplementary Fig. 3). This suggests that aberrant light exposure drives altered cardiac function. Due to the altered phase of entrainment, both 22.5 and $27 \mathrm{~h} \mathrm{LD}$ housed mice are exposed to light at inappropriate times (relative to their internal rhythm). Therefore, we assessed the effect of acute mistimed light exposure on HR by exposing control $24 \mathrm{~h}$ LD housed mice to a $2 \mathrm{~h}$ light pulse in the early active (dark) phase (ZT14-16). As expected, light exposure caused a significant reduction in activity, $T_{\mathrm{b}}$ and HR. However, unlike $T_{\mathrm{b}}$ and activity, mistimed light caused a profound decrease in HR that remained depressed in light-pulsed mice for a further two LD cycles as the mice regained normal phase alignment to the LD cycle (Fig. 5i, j; Supplementary Fig. 4).

Fig. 3 Robust circadian rhythms in clock gene expression are maintained in non-24 h LD housed mice. Representative brain sections a and quantification $\mathbf{b}$ of radioactive in situ hybridisation for Per1 expression in the SCN from mice housed in $24 \mathrm{~h}$ (black) or $22.5 \mathrm{~h}$ (red) conditions for 17 week. c Circulating corticosterone profiles were delayed relative to the LD cycle in mice housed under or $22.5 \mathrm{~h}$ conditions. $\mathbf{d}$ Acrophase analysis of Per 1 and corticosterone rhythms in 24 and $22.5 \mathrm{~h}$ housed mice reveals consistent phase delay. e Profiling of clock gene expression in peripheral tissues of mice housed in 24 or $22.5 \mathrm{~h}$ LD conditions for 17 weeks. f Acrophase analyses across genes and tissues (plotted relative to respective light cycle) demonstrates that synchronisation both within and across tissue clocks is maintained in non-24 $\mathrm{h}$ LD conditions. All data plotted mean \pm SEM relative to the respective light cycle ( 24 or $22.5 \mathrm{~h}$ ) and normalised to ZTO of the $24 \mathrm{~h}$ LD group $(n=4 /$ time-point/group). *Significant $(p<0.05)$ difference in phase between 24 and $22.5 \mathrm{~h}$ profiles; a significant difference in amplitude or mesor between 24 and $22.5 \mathrm{~h}$ LD conditions (sinusoidal waveform fits with $\mathrm{F}$ tests for shared characteristics). Scale bar in a $=3 \mathrm{~mm}$ 

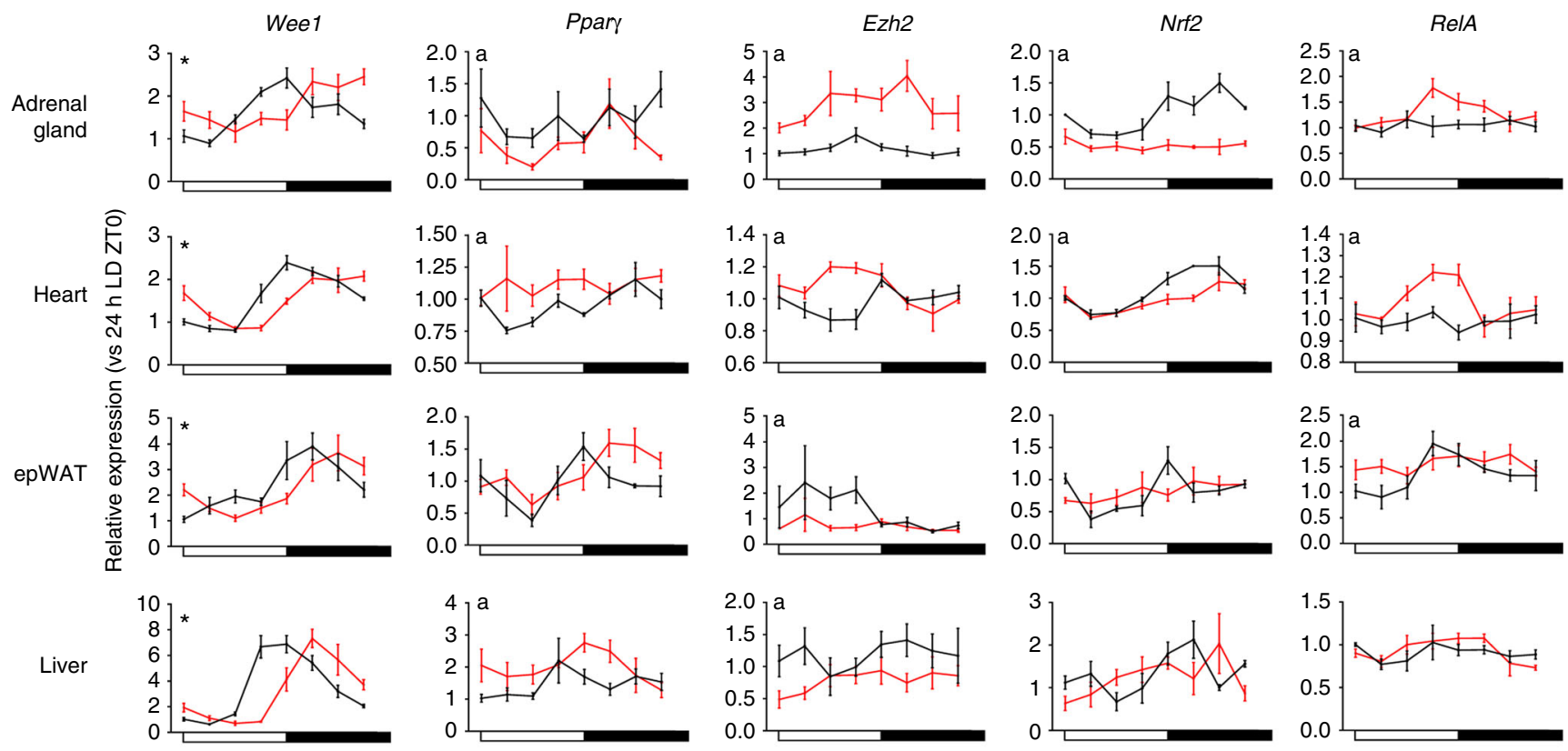

$-24 \mathrm{~h} \mathrm{LD}$

$-22.5 \mathrm{~h} \mathrm{LD}$

Fig. 4 Phase misalignment disrupts rhythmic gene expression in a pathways and tissue-specific manner. Transcript profiles of major clock-controlled regulator genes of cell cycle (Wee1), epigenetic regulation (Ezh2), metabolism (Ppary), oxidative stress (Nrf2) and inflammation (RelA) from mice housed in 24 or $22.5 \mathrm{~h}$ conditions for 17 weeks. All data plotted mean \pm SEM relative to the respective light cycle ( 24 or $22.5 \mathrm{~h}$ ) and normalised to ZTO of the $24 \mathrm{~h} \mathrm{LD}$ group ( $n=4$ /time-point/group). *Significant $(p<0.05)$ difference in phase between 24 and $22.5 \mathrm{~h}$ profiles; a = significant difference in amplitude or mesor between 24 and $22.5 \mathrm{~h} \mathrm{LD}$ conditions (sinusoidal waveform fits with $\mathrm{F}$ tests for shared characteristics)

HR can be acutely affected by environmental light via autonomic signalling 36,37 . Our studies suggest that suppression of cardiac function and lengthening of QT, results from discordance between the phase of the local clockwork and extrinsic signals such as altered autonomic input to the heart. We tested this directly in 22.5 and $24 \mathrm{~h} \mathrm{LD}$ housed mice by blocking vagal and sympathetic input to the heart using atropine/propranolol administration ${ }^{38-40}$. The impact of autonomic block on HR was assessed in both the active ( $2 \mathrm{~h}$ after lights on; $\sim \mathrm{ZT} 2)$ and inactive ( $2 \mathrm{~h}$ after lights off, ZT14) phases of the cycle under both lighting conditions. Indeed, at both time points, complete autonomic blockade caused a significant increase in HR under $22.5 \mathrm{~h} \mathrm{LD}$, but not in $24 \mathrm{~h} \mathrm{LD}$ housed mice and resulted in comparable HR in both groups (Fig. 5k-m). Thus, our findings clearly demonstrate that discordance between environmental light/dark cycles and the internal clockwork cause a rapid and profound depression of cardiac function, including prolonged PR and QT interval. This effect is independent of locomotor activity, and is mediated by altered autonomic signalling.

\section{Discussion}

These studies provide direct evidence that chronic desynchrony between the internal circadian clock and environmental light cycles profoundly impacts mammalian physiology. Housing mice under non- $24 \mathrm{~h} \mathrm{LD}$ cycles created an experimental paradigm, which mimics the phase misalignment experienced by humans with extreme chronotype and those engaged in shift work. Despite achieving stable entrainment, phase misalignment had a rapid impact on energy metabolism and cardiac rate and conduction, which did not diminish over many months. Remarkably, dysregulated metabolic and cardiac profiles were not driven by disruption of the molecular clockwork, nor by a loss of phase synchrony between different tissue oscillators (internal desynchrony). Instead, our studies clearly indicate that it is the altered phase relationship between the external light cycle and internal circadian timing that drives the aberrant physiology.

Many animal studies have inferred the importance of circadian timing for maintaining a healthy physiological state through genetic disruption of one or more components of the underlying molecular clock. However, this approach rarely allows the importance of altered timing per se to be determined due to constitutive loss of the factor and its pleiotropic actions ${ }^{19}$. In contrast, resonance studies, as we use here, exploit the altered interaction between an intact biological clock and its external environment. The adaptive benefit of matching internal circadian time to environmental cycles has been robustly demonstrated in lower organisms ${ }^{18}$, and a few studies have demonstrated that altered circadian timing can reduce survival fitness in natural settings ${ }^{41,42}$. Yet few studies have used circadian resonance to reveal the impact of circadian timing to internal physiology and health in mammalian models. Here we show that under non- $24 \mathrm{~h}$ LD cycles, mice exhibited markedly reduced energy efficiency and increased reliance on carbohydrate energy substrates. This finding reinforces the long held assertion that circadian timing serves to optimise response to the environment, including optimising cycles of energy storage and mobilisation. Decreased energy efficiency was at least in part due to increased energy expenditure in the $22.5 \mathrm{~h} \mathrm{LD}$ housed mice. Phase misalignment may also lead to reduced nutrient absorption and/or utilisation as both are influenced by the circadian system $^{43}$. In a notable study, Martino and colleagues reported that short period CK1 $\varepsilon^{\text {taul }+}$ mutant hamsters (endogenous period of $\sim 22 \mathrm{~h}$ ) were prone to cardio-renal dysfunction when housed under $24 \mathrm{~h} \mathrm{LD}$ cycles, which did not manifest if the animals were maintained on a $22 \mathrm{~h} \mathrm{LD} \mathrm{cycle}{ }^{44}$. In agreement with our studies, this indicates that an altered phase of entrainment is sufficient to drive pathology, and highlights a particular vulnerability of the cardiovascular system. 
In pursuit of further consequences of environmental desynchrony, we profiled clock output genes across tissues. These studies showed tissue specific, and gene specific patterns of dysregulation, with enrichment for specific pathways (notably oxidative stress response in the heart and adrenal gland). Disruption of rhythmic transcription despite robust circadian clock gene oscillation has been demonstrated previously in both mice and humans ${ }^{45-47}$, indicating a de-coupling possibly due to mistimed external signals. Moreover, it is becoming clear that rhythmic processes within a tissue can be remodelled and detached from the circadian clock under environmental or pathological challenge ${ }^{48,49}$. The altered expression of epigenetic regulators (e.g. EZH2, TET enzymes) in response to non-24 LD schedules, is strikingly similar to that observed in the SCN of $22 \mathrm{~h}$ LD housed mice reported by Brown and colleagues ${ }^{45}$, and suggests that epigenetic mechanisms drive reprogramming of clock-controlled processes during phase misalignment. a

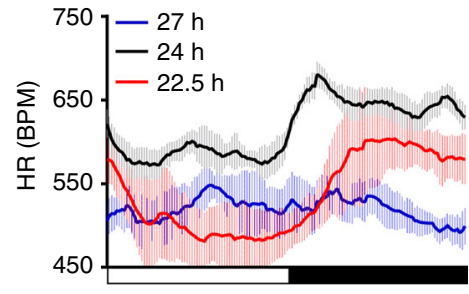

d

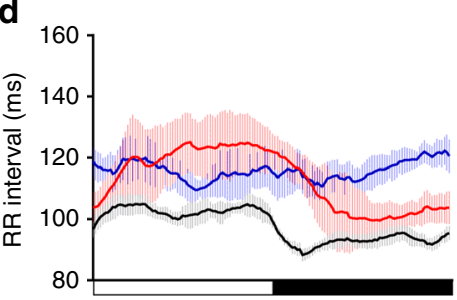

f

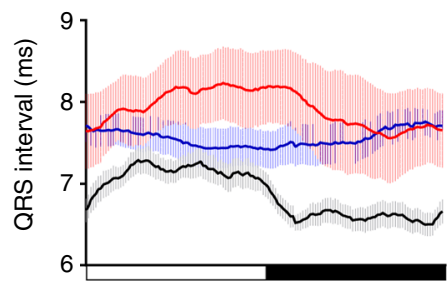

h

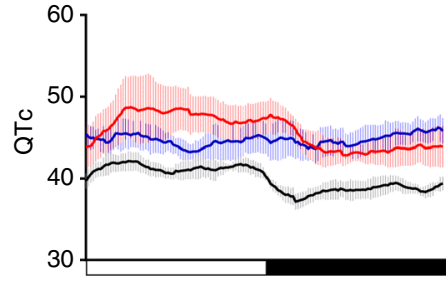

b
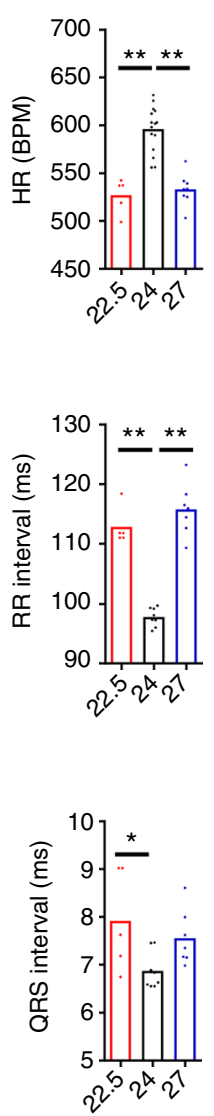

e

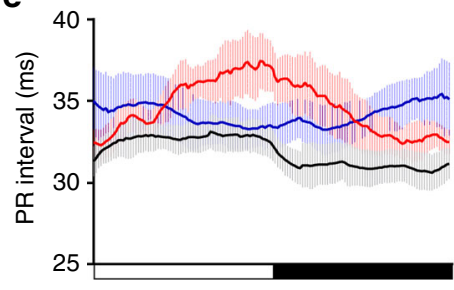

g

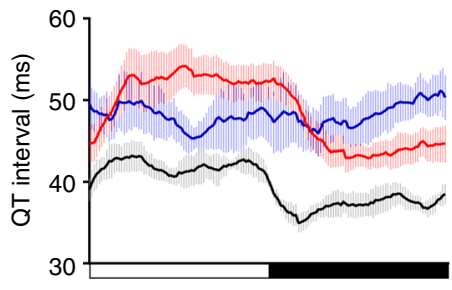

i

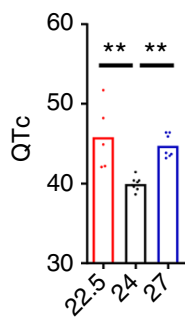

C
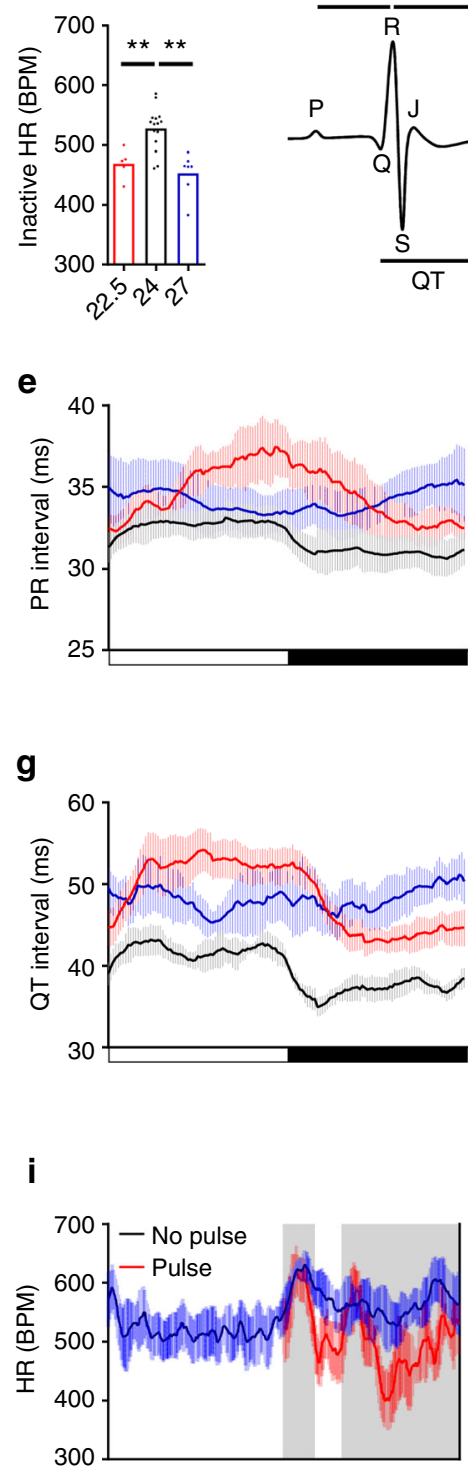
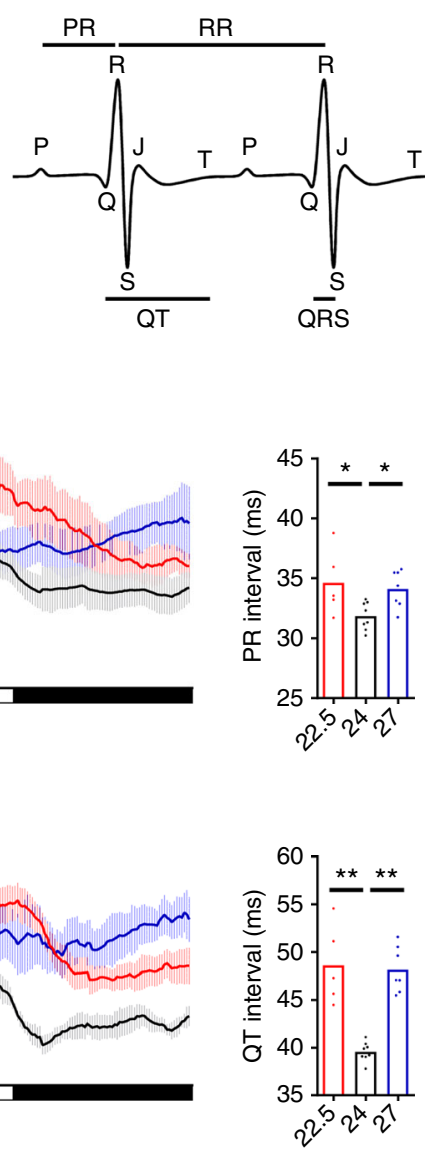

j

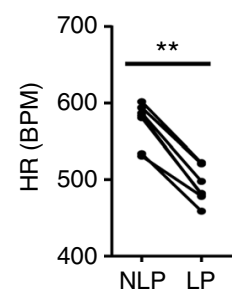

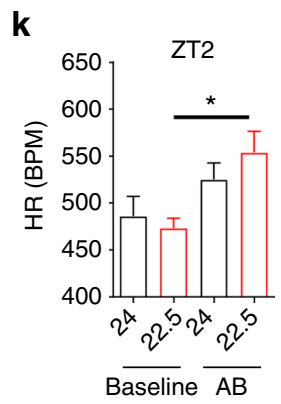

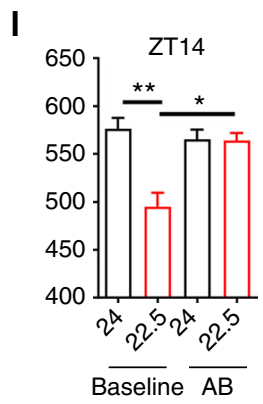

m

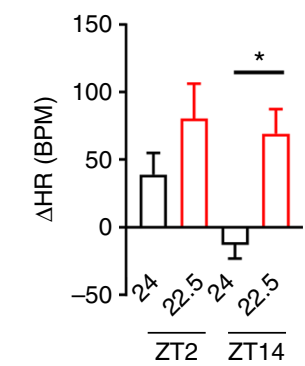


Cardiac physiology exhibits pronounced circadian rhythmicity, driven both by the local cardiac clock and through circadian variation in autonomic input to the heart ${ }^{32,33,58-53}$. In our studies, control mice exhibited robust rhythms in ECG parameters including RR, PR and QTc. All of these parameters were significantly prolonged when mice were placed under non-24 LD cycles. Altered cardiac conduction is associated with severe pathology and sudden cardiac death, and is therefore of profound interest to human health. The incidence of sudden cardiac death follows a strong diurnal rhythm, with peak incidence around the transition from sleep to wake ${ }^{54}$, 55; and increased incidence of heart disease and cardiac events is associated with nightshift workers ${ }^{56}$. Our results indicate that mistimed light drives inappropriate autonomic input to the heart, and suggests that circadian misalignment increases vulnerability to conduction block and ventricular arrhythmia (due to significant lengthening of PR and QTc). The dampening of the central SCN circadian oscillator observed in the non-resonant housed mice may weaken normal circadian control over autonomic output to the heart and other tissues, and thereby exacerbate phase misalignment between the local tissue clocks and autonomic drive.

Remarkably, depression of HR was observed in response to acute mistimed light exposure, which persisted over several days. The detrimental impact of mistimed light is clear, and is supported by reports of depressed HR and prolonged QT in mice housed under constant light or subject to light-restricted feeding ${ }^{57}$. Cardiomyocyte-specific deletion of Bmall leads to a similar lengthening of QRS and QTc intervals, which was ascribed to aberrant expression of cardiac ion channels $\operatorname{Scn} 5 a$ and $K c n h 2^{58,59}$. We did not observe altered expression of $\operatorname{Scn} 5 a$, Kcnh2, or a number of other ion channels in cardiac tissue of $22.5 \mathrm{~h}$ housed mice. We show that autonomic block can normalise HR in non-resonant LD housed mice, demonstrating an underlying role for inappropriate autonomic signalling during phase misalignment with the LD cycle. In contrast to the mice, acute light exposure increases HR in humans. Nevertheless, important parallels exist between rodent and human HR/ECG responses to altered lighting/circadian desynchrony. Rats and humans exhibit a similar diurnal variation in the fractal structure of HR (a measure of HR variability), despite the opposite nature of diurnal/nocturnal activity in the species ${ }^{60}$. Most importantly, altered autonomic signalling and long QTc interval have been consistently reported in studies of shift work in humans (e.g. refs ${ }^{61-63}$ ). Thus, in both humans and mice, the strong influence of both extrinsic (autonomic) and intrinsic (local cardiac clock) timing signal to cardiac pacing is likely to increase the vulnerability of the cardiovascular system to dysfunction under conditions of circadian disruption.

By employing non-resonant light cycles, our studies isolate the physiological impact of circadian misalignment in a mammalian model. Given that the phase misalignment produced in our studies mimic the impact of extreme chronotype and shift work on circadian phase in humans ${ }^{22,64-66}$, our findings provide important new insight into human pathologies associated with shift work, chronotype and social jetlag.

\section{Methods}

Animals. All animal experiments were licenced under the Animals (Scientific Procedures) Act of 1986 (UK), and conducted in accordance with University of Manchester animal welfare committee guidelines. Male C57Bl/6J mice were purchased from Charles River (UK), and $C k 1 \varepsilon^{t a u}$ mice ${ }^{67}$ were bred at the University of Manchester. At 8 weeks of age, male mice were assigned randomly to each LD condition and transferred into light-tight housing cabinets and maintained in $20,22.5,24,27$ or $30 \mathrm{~h}$ LD cycles for 17-52 weeks. Light levels were maintained at 463 lux during the light phase and during light pulse experiments. Ambient temperature was $22 \pm 2{ }^{\circ} \mathrm{C}$, with food and water available ad libitum. Mice remained group housed throughout, except where single housing was necessitated by physiological monitoring (e.g. CLAMS, ECG telemetry, food intake recording) Due to different lighting schedules, blinding of the experimenter to experimental conditions was not possible.

Behavioural and physiological monitoring. To assess body composition, whole body lean and fat mass was assessed using the EchoMRI system (Echo Medical Systems). For long-term ( $>10$ week) recording of body temperature, mice were implanted with iButton temperature loggers (Maxim, DS1922L-F5). Implants were de-housed, programmed and encapsulated in a $20 \%$ Poly(ethylene-co-vinyl acetate) and $80 \%$ paraffin mixture ${ }^{68}$ before implantation into the peritoneal cavity (ip). Temperature recordings were calibrated by immersion of the iButtons into set temperature water baths before implantation. In separate experiments, $T_{\mathrm{b}}$ and locomotor activity rhythms were recorded using indwelling radio-telemetry devices (TA-F10, DSI international) implanted ip. Continuous measures of food intake were recorded from singly housed animals using PhenoMaster behavioural cages (TSE Systems). Mice ( $>8$ week LD cycle exposure) were acclimatised to the cages for two cycles, after which food consumption was measured in 5 min bins for 3-4 LD cycles. To assess metabolic gas exchange, mice were individually housed in indirect calorimetry cages (CLAMS, Columbus Instruments). As above, mice (>8 week LD cycle exposure) were acclimatised to the cages for two cycles, following which $\mathrm{O}_{2}$ consumption and $\mathrm{CO}_{2}$ production were recorded every $10 \mathrm{~min}$ for $>3 \mathrm{LD}$ cycles. RER was derived from these measures $\left(\mathrm{VCO}_{2} / \mathrm{VO}_{2}\right)$, as were protein oxidation independent $\mathrm{COx}\left(4.55^{*} \mathrm{VCO}_{2}-3.21^{*} \mathrm{VO}_{2}\right)^{69}$ and energy expenditure $\left(3.815 * \mathrm{VO}_{2}+1.232 * \mathrm{VCO}_{2}\right)$.

Heart rate and ECG recording. Mice were implanted with ETA-F10 radio-telemetry devices (Data sciences international) for recording of locomotor activity, $T_{\mathrm{b}}$ and ECG. Devices were implanted ip with ECG recording leads brought through the abdominal wall and negative lead secured $\sim 1 \mathrm{~cm}$ right of midline at upper chest, positive lead secured $\sim 1 \mathrm{~cm}$ to the left of midline at the xiphoid plexus. Mice recovered for 7-10 days before the start of recording the experiment. Mice were excluded from the study if lead placement was not maintained throughout the experiment. For longitudinal studies (i.e. $>7$ days), activity, $T_{\mathrm{b}}$ and $10 \mathrm{~s}$ ECG data sweeps were collected every $10 \mathrm{~min}$, and a minimum of three consecutive days used for each analyses period. 'Inactive heart rate' was defined as periods where no activity had been recorded for $>20 \mathrm{~min}$ before the ECG sweep recording. Following recording, ECG waveforms (10 s sweeps at 1 sweep/10 min) were processed and analysed using bespoke software programme written in Matlab (Mathworks). In brief, ECG R waves (local maxima) were extracted by amplitude windowing (typically $0.3-3 \mathrm{~V}$ ) and subsequent template matching to the mean beat waveform. For this analysis, we calculated an ECG waveform template for each individual sweep (the normalised mean of all beats within a pre-defined amplitude window) which then served to identify and remove occasional spurious threshold crossings due to noise. Individual recording sweeps were excluded from subsequent analysis where the mean amplitude of discriminated beat waveforms was $<3$ times the lower limit of the amplitude window, where baseline variation in the ECG trace exceed $1 / 3$ of that lower limit and/or where $>20 \%$ of the events detected were excluded by the template matching algorithm. Specific ECG parameters (RR, PR,

Fig. 5 Misalignment with environmental LD cycles disrupts cardiac rate and leads to long QT interval. a Incomparison to matched $24 \mathrm{~h}$ LD cycle housed mice, mean heart rate (HR) was significantly reduced in mice maintained in 22.5 and $27 \mathrm{~h}$ LD cycles, which was accompanied by a significant reduction in the amplitude of HR rhythms in the $27 \mathrm{~h}$ LD mice. $\mathbf{b}$ HR remained significantly reduced in non-24 $\mathrm{h}$ LD housed mice even when analyses were limited to periods of inactive (no recorded movement for 20 min preceding HR measure). c Murine ECG beat waveform with defining features labelled. $\mathbf{d}-\mathbf{h}$ Diurnal profiles and mean interval durations for RR (d), PR (e), QRS (f), QT (g) and RR-adjusted QT (QTc; $\mathbf{h}$ ) of mice maintained under $24 \mathrm{~h}, 22.5 \mathrm{~h}$ or $27 \mathrm{~h}$ LD conditions. All parameters were significantly lengthened under non-24 h LD cycles. $\mathbf{i}, \mathbf{j}$ A $2 \mathrm{~h}$ light pulse (LP, red) from ZT14-16 caused a significant reduction in night-time HR when compared with the average HR profile recorded over three preceding cycles (blue, NLP). k-m Complete autonomic blockade (AB) increased HR at ZT2 ( 2 h after lights on; $\mathbf{k})$ and ZT14 ( $2 \mathrm{~h}$ after lights off; $\mathbf{I})$ in $22.5 \mathrm{~h}$ LD housed mice, and normalised it to $24 \mathrm{~h}$ LD conditions. $\mathbf{m}$ Change in HR (vs. baseline) during autonomic blockade at ZT2 and ZT14. Diurnal profiles depict mean $\pm 95 \% \mathrm{Cl}$. ${ }^{*} p<0.05, * * p<0.01$. a-h One-way ANOVA with Dunnett's post hoc test (vs. $24 \mathrm{~h}$ control group); j paired $t$-test; $\mathbf{k}-\mathbf{m}$ repeated measures two-way ANOVA with Tukey's post hoc test 
QRS, QT) were then collected for each beat by automated identification and recording of appropriate deflection points between each valid R-R interval in the trace. Within our data sets $>80 \%$ of analysed traces passed this quality control step and, as a matter of routine, we manually inspected randomly chosen subsets of valid and excluded traces to confirm appropriate categorisation and analysis. Importantly, we found that using more or less stringent quality control parameters did not affect the overall results of our subsequent analysis, nor did we find any bias with respect to the proportion of traces excluded from analysis during active vs. inactive portions of the daily cycle. For autonomic blockade, conscious free-moving $22.5 \mathrm{~h}$ and $24 \mathrm{~h} \mathrm{LD}$ housed mice were injected either $2 \mathrm{~h}$ after lights on ( ZT2) or $2 \mathrm{~h}$ after lights off ( ZT14) with atropine $(0.5 \mathrm{mg} / \mathrm{kg}$, ip) followed by propranolol ( $1 \mathrm{mg} / \mathrm{kg}$, ip). During autonomic blockade studies, radio-telemetry data (activity, $T_{\mathrm{b}}$ and $\mathrm{HR}$ ) was collected every minute. Baseline HR was derived from $1 \mathrm{~h}$ pre-injection, with HR during complete autonomic blockade collected 20-30 min post-administration. Injection-induced increases in HR following vehicle administration (conducted 1 cycle before autonomic blockade) returned to baseline by $15 \mathrm{~min}$ post-injection.

Gene expression analyses. For qPCR, tissues were rapidly dissected and snap frozen at 8 time-points spaced equally through the 24 and $22.5 \mathrm{~h} \mathrm{LD}$ cycles. Total RNA was extracted using Trizol reagent (Life Technologies) according with the manufacturer's protocol. cDNA was synthesised using High capacity RNA to cDNA kit (Applied Biosystems). qPCR was performed using GoTaq Master Mix (Promega) and an applied biosystems 7900384 well thermal cycler (Applied Biosystems). Relative gene expression was quantified using the $\Delta \Delta \mathrm{CT}$ method using Ppib and $r 18$ s as reference genes. Primer sequences are listed in Supplementary Table 1. For in situ hybridisation, brains were frozen over dry ice, cryo-sectioned $(12 \mu \mathrm{m})$, mounted onto poly-L slides, and stored at $-80^{\circ} \mathrm{C}$. Per 1 plasmid was a kind gift from Prof Urs Albrecht (University of Freibourg). The cDNA fragment was cloned into a pGEMT-easy vector (Promega) and linearised using BamHI and XhoI to produce sense and antisense templates, respectively. Riboprobes were synthesised in the presence of 33UTP, and hybridised overnight at $60^{\circ} \mathrm{C}$. Hybridisation was visualised by exposing autoradiographic film at $-80^{\circ} \mathrm{C}$ for 7 days. Signal intensity was quantified by densitometry analysis of SCN region normalised to background staining. Normalised optical density for the SCN reflects $>3$ sections per animal and 3-4 animals/tp

Statistical analyses. Experimental design and $n$ number determination was based on previous experience and appropriate power analyses $(\alpha=0.05,80 \%$ power, $20-50 \%$ estimated effect size depending on measure). Data are presented as mean \pm SEM or $95 \% \mathrm{CI}$ as indicated. Mean difference comparisons were carried out using Student's $t$-test (two-sided; paired where appropriate), one-way ANOVA, and two-way ANOVA with post hoc tests and repeated measures as appropriate. Determination of acrophase in clock gene expression (Fig. 3) was performed by harmonic regression using CircWave v1.4 software so $^{70}$. For statistical comparison of rhythmic characteristics (mesor, phase, amplitude) of gene expression and corticosterone profiles, best-fit sinusoidal waveforms were generated by regression analyses and shared characteristics tested with equal sum of square $F$ tests (Graphpad Prism 7.0). For longitudinal measures (e.g. HR), sinusoidal waveforms were generated for each individual, from which rhythm characteristics were derived and group mean differences tested with one-way ANOVA.

Data availability. Materials, algorithms and data generated in these studies are available from the authors upon reasonable request.

Received: 21 December 2016 Accepted: 30 June 2017

Published online: 12 September 2017

\section{References}

1. Lamia, K. A., Storch, K. F. \& Weitz, C. J. Physiological significance of a peripheral tissue circadian clock. Proc. Natl Acad. Sci. USA 105, 15172-15177 (2008).

2. Marcheva, B. et al. Disruption of the clock components CLOCK and BMAL1 leads to hypoinsulinaemia and diabetes. Nature 466, 627-631 (2010).

3. Dyar, K. A. et al. Muscle insulin sensitivity and glucose metabolism are controlled by the intrinsic muscle clock (vol 3, pg 29, 2014). Mol. Metab. 3, 857-857 (2014)

4. Carter, S. J. et al. A matter of time: study of circadian clocks and their role in inflammation. J. Leukoc. Biol. 99, 549-560 (2016).

5. Alibhai, F. J., Tsimakouridze, E. V., Reitz, C. J., Pyle, W. G. \& Martino, T. A. Consequences of circadian and sleep disturbances for the cardiovascular system. Can. J. Cardiol. 31, 860-872 (2015).

6. Litinski, M., Scheer, F. A. \& Shea, S. A. Influence of the circadian system on disease severity. Sleep Med. Clin. 4, 143-163 (2009).
7. Leproult, R., Holmback, U. \& Van Cauter, E. Circadian misalignment augment markers of insulin resistance and inflammation, independently of sleep loss. Diabetes 63, 1860-1869 (2014).

8. Young, M. E. \& Bray, M. S. Potential role for peripheral circadian clock dyssynchrony in the pathogenesis of cardiovascular dysfunction. Sleep Med. 8, 656-667 (2007).

9. Morris, C. J., Purvis, T. E., Hu, K. \& Scheer, F. A. Circadian misalignment increases cardiovascular disease risk factors in humans. Proc. Natl Acad. Sci. USA 113, E1402-E1411 (2016).

10. Lane, J. M. et al. Genome-wide association analysis identifies novel loci for chronotype in 100,420 individuals from the UK Biobank. Nat. Commun. 7, 10889 (2016)

11. Jones, S. E. et al. Genome-wide association analyses in 128,266 individuals identifies new morningness and sleep duration loci. PLoS Genet. 12, e1006125 (2016).

12. Arora, T. \& Taheri, S. Associations between late chronotype, body mass index and dietary behaviors in young adolescents. Int. J. Obes. 39, 39-44 (2014).

13. Reutrakul, S. \& Van Cauter, E. Interactions between sleep, circadian function, and glucose metabolism: implications for risk and severity of diabetes. Ann. N. Y. Acad. Sci. 1311, 151-173 (2014)

14. Barnard, A. R. \& Nolan, P. M. When clocks go bad: neurobehavioural consequences of disrupted circadian timing. PLoS Genet. 4, e1000040 (2008).

15. Bechtold, D. A., Gibbs, J. E. \& Loudon, A. S. Circadian dysfunction in disease Trends Pharmacol. Sci. 31, 191-198 (2010).

16. Fu, L., Patel, M. S., Bradley, A., Wagner, E. F. \& Karsenty, G. The molecular clock mediates leptin-regulated bone formation. Cell 122, 803-815 (2005).

17. Scheiermann, C., Kunisaki, Y. \& Frenette, P. S. Circadian control of the immune system. Nat. Rev. Immunol. 13, 190-198 (2013).

18. West, A. C. \& Bechtold, D. A. The cost of circadian desynchrony: Evidence, insights and open questions. Bioessays 37, 777-788 (2015).

19. Yang, G. R. et al. Timing of expression of the core clock gene Bmall influences its effects on aging and survival. Sci. Transl. Med. 8, 324ra16 (2016).

20. Wright, K. P., Gronfier, C., Duffy, J. E. \& Czeisler, C. A. Intrinsic period and light intensity determine the phase relationship between melatonin and sleep in humans. J. Biol. Rhythms 20, 168-177 (2005).

21. Wyatt, J. K., Dijk, D. J., Ritz-De Cecco, A., Ronda, J. M. \& Czeisler, C. A. Sleep-facilitating effect of exogenous melatonin in healthy young men and women is circadian-phase dependent. Sleep 29, 609-618 (2006).

22. Wright, K. P., Dinges, D. F., Roth, T., Walsh, J. K. \& Czeisler, C. A. Circadian phase in patients with shift-work disorder (Swd): influence on nightime sleepiness, performance and daytime sleep. Sleep 32, A46-A46 (2009).

23. Bechtold, D. A. Energy-responsive timekeeping. J. Genet. 87, 447-458 (2008)

24. Perelis, M., Ramsey, K. M. \& Bass, J. The molecular clock as a metabolic rheostat. Diabetes Obes. Metab. 17, 99-105 (2015).

25. Green, C. B., Takahashi, J. S. \& Bass, J. The meter of metabolism. Cell 134 728-742 (2008)

26. Kalsbeek, A. et al. Circadian rhythms in the hypothalamo-pituitary-adrenal (HPA) axis. Mol. Cell Endo. 349, 20-29 (2012)

27. Grimaldi, B. et al. PER2 controls lipid metabolism by direct regulation of PPARgamma. Cell Metab. 12, 509-520 (2010).

28. Matsuo, T. et al. Control mechanism of the circadian clock for timing of cell division in vivo. Science 302, 255-259 (2003)

29. Pekovic-Vaughan, V. et al. The circadian clock regulates rhythmic activation of the NRF2/glutathione-mediated antioxidant defense pathway to modulate pulmonary fibrosis. Gene Dev. 28, 548-560 (2014).

30. Spengler, M. L. et al. Core circadian protein CLOCK is a positive regulator of NF-kappa B-mediated transcription. Proc. Natl Acad. Sci. USA 109 E2457-E2465 (2012)

31. Etchegaray, J. P. et al. The polycomb group protein EZH2 is required for mammalian circadian clock function. J. Biol. Chem. 281, 21209-21215 (2006)

32. Martino, T. et al. Day/night rhythms in gene expression of the normal murine heart. J. Mol. Med. 82, 256-264 (2004).

33. Guo, Y. F. \& Stein, P. K. Circadian rhythm in the cardiovascular system: chronocardiology. Am. Heart. J. 145, 779-786 (2003).

34. Scheer, F. A., Hilton, M. F., Mantzoros, C. S. \& Shea, S. A. Adverse metabolic and cardiovascular consequences of circadian misalignment. Proc. Natl Acad. Sci. USA 106, 4453-4458 (2009).

35. Hedley, P. L. et al. The genetic basis of long QT and short QT syndromes: a mutation update. Hum. Mutat. 30, 1486-1511 (2009).

36. Scheer, F. A. J. L., van Doornen, L. J. P. \& Buijs, R. M. Light and diurnal cycle affect autonomic cardiac balance in human; poss ible role for the biological clock. Auton. Neurosci-Basic 110, 44-48 (2004).

37. Schroeder, A., Loh, D. H., Jordan, M. C., Roos, K. P. \& Colwell, C. S. Circadian regulation of cardiovascular function: a role for vasoactive intestinal peptide. Am. J. Physiol. Heart Circ. Phys. 300, H241-H250 (2011).

38. Leoni, A. L. et al. Chronic heart rate reduction remodels ion channel transcripts in the mouse sinoatrial node but not in the ventricle. Physiol. Genomics 24, 4-12 (2005). 
39. Gehrmann, J. et al. Electrophysiological characterization of murine myocardial ischemia and infarction. Basic Res. Cardiol. 96, 237-250 (2001).

40. Berul, C. I. et al. Ventricular arrhythmia vulnerability in cardiomyopathic mice with homozygous mutant myosin-binding protein C gene. Circulation 104, 2734-2739 (2001).

41. DeCoursey, P. J. \& Krulas, J. R. Behavior of SCN-lesioned chipmunks in natural habitat: a pilot study. J. Biol. Rhythms 13, 229-244 (1998).

42. Spoelstra, K., Wikelski, M., Daan, S., Loudon, A. S. \& Hau, M. Natural selection against a circadian clock gene mutation in mice. Proc. Natl Acad. Sci. USA 113, 686-691 (2016).

43. Stenvers, D. J., Jonkers, C. F., Fliers, E., Bisschop, P. H. \& Kalsbeek, A. Nutrition and the circadian timing system. Prog. Brain. Res. 199, 359-376 (2012).

44. Martino, T. A. et al. Circadian rhythm disorganization produces profound cardiovascular and renal disease in hamsters. Am. J. Physiol-Reg I 294, R1675-R1683 (2008).

45. Azzi, A. et al. Circadian behavior is light-reprogrammed by plastic DNA methylation. Nat. Neurosci. 17, 377-382 (2014).

46. Archer, S. N. et al. Mistimed sleep disrupts circadian regulation of the human transcriptome. Proc. Natl Acad. Sci. USA 111, E682-E691 (2014).

47. Moller-Levet, C. S. et al. Effects of insufficient sleep on circadian rhythmicity and expression amplitude of the human blood transcriptome. Proc. Natl Acad. Sci. USA 110, E1132-E1141 (2013).

48. Masri, S. et al. Lung adenocarcinoma distally rewires hepatic circadian homeostasis. Cell 165, 896-909 (2016).

49. Eckel-Mahan, K. L. et al. Reprogramming of the circadian clock by nutritional challenge. Cell 155, 1464-1478 (2013)

50. Scheer, F. A., Kalsbeek, A. \& Buijs, R. M. Cardiovascular control by the suprachiasmatic nucleus: neural and neuroendocrine mechanisms in human and rat. Biol. Chem. 384, 697-709 (2003).

51. Scheer, F. A. et al. Impact of the human circadian system, exercise, and their interaction on cardiovascular function. Proc. Natl Acad. Sci. USA 107, 20541-20546 (2010)

52. Young, M. E. et al. Cardiomyocyte-specific BMAL1 plays critical roles in metabolism, signaling, and maintenance of contractile function of the heart. J. Biol. Rhythms 29, 257-276 (2014)

53. Martino, T. A. \& Young, M. E. Influence of the cardiomyocyte circadian clock on cardiac physiology and pathophysiology. J. Biol. Rhythms 30, 183-205 (2015).

54. Muller, J. E. et al. Circadian variation in the frequency of sudden cardiac death. Circulation 75, 131-138 (1987).

55. Jeyaraj, D. et al. Circadian rhythms govern cardiac repolarization and arrhythmogenesis. Nature 483, 96-U141 (2012).

56. Knutsson, A. Health disorders of shift workers. Occupational Med. 53, 103-108 (2003).

57. Schroder, E. A. et al. Light phase-restricted feeding slows basal heart rate to exaggerate the type-3 long QT syndrome phenotype in mice. Am. J. Physiol.Heart C 307, H1777-H1785 (2014).

58. Schroder, E. A. et al. The cardiomyocyte molecular clock, regulation of Scn5a, and arrhythmia susceptibility. Am. I. Physiol.-Cell Ph 304, C954-C965 (2013).

59. Schroder, E. A. et al. The cardiomyocyte molecular clock regulates the circadian expression of Kcnh2 and contributes to ventricular repolarization. Heart Rhythm 12, 1306-1314 (2015).

60. Hu, K., Scheer, F. A., Buijs, R. M. \& Shea, S. A. The circadian pacemaker generates similar circadian rhythms in the fractal structure of heart rate in humans and rats. Cardiovasc. Res. 80, 62-68 (2008).

61. Murata, K., Yano, E. \& Shinozaki, T. Impact of shift work on cardiovascular functions in a 10-year follow-up study. Scand. J. Work. Environ. Health. 25, 272-277 (1999)

62. Ishii, N., Dakeishi, M., Sasaki, M., Iwata, T. \& Murata, K. Cardiac autonomic imbalance in female nurses with shift work. Auton. Neurosci. 122, 94-99 (2005).

63. Furlan, R. et al. Modifications of cardiac autonomic profile associated with a shift schedule of work. Circulation 102, 1912-1916 (2000).
64. Czeisler, C. A. et al. Exposure to bright light and darkness to treat physiologic maladaptation to night work. N. Engl. J. Med. 322, 1253-1259 (1990).

65. Santhi, N., Duffy, J. F., Horowitz, T. S. \& Czeisler, C. A. Scheduling of sleep/darkness affects the circadian phase of night shift workers. Neurosci. Lett 384, 316-320 (2005).

66. Smith, M. R., Cullnan, E. E. \& Eastman, C. I. Shaping the light/dark pattern for circadian adaptation to night shift work. Physiol. Behav. 95, 449-456 (2008).

67. Meng, Q. J. et al. Setting clock speed in mammals: the CK1 epsilon tau mutation in mice accelerates circadian pacemakers by selectively destabilizing PERIOD proteins. Neuron 58, 78-88 (2008).

68. Lovegrove, B. G. Modification and miniaturization of Thermochron iButtons for surgical implantation into small animals. J. Comp. Physiol. B 179, 451-458 (2009).

69. Frayn, K. N. Calculation of substrate oxidation rates in vivo from gaseous exchange. J. Appl. Physiol. Respir. Environ. Exerc. Physiol. 55, 628-634 (1983).

70. Oster, H., Damerow, S., Hut, R. A. \& Eichele, G. Transcriptional profiling in the adrenal gland reveals circadian regulation of hormone biosynthesis genes and nucleosome assembly genes. J. Biol. Rhythms 21, 350-361 (2006).

\section{Acknowledgements}

We thank the Biological Services Unit at the University of Manchester for support of in vivo studies. This work was supported by the Biotechnology and Biological Sciences Research Council (UK) through grants to D.A.B. (BB/I01864/1; BB/J017744/1) and T.M.B. (BB/N007115/1)

\section{Author contributions}

All authors contributed to experimental design; A.C.W., L.S. and D.A.B. conducted experiments; A.C.W., T.M.B. and D.A.B. analyzed results; A.C.W., D.W.R., A.S.I.L., T.M. B. and D.A.B. wrote the manuscript; D.A.B. conceived the project and supervised all aspects.

\section{Additional information}

Supplementary Information accompanies this paper at doi:10.1038/s41467-017-00462-2.

Competing interests: The authors declare no competing financial interests.

Reprints and permission information is available online at http://npg.nature.com/ reprintsandpermissions/

Publisher's note: Springer Nature remains neutral with regard to jurisdictional claims in published maps and institutional affiliations.

Open Access This article is licensed under a Creative Commons Attribution 4.0 International License, which permits use, sharing, adaptation, distribution and reproduction in any medium or format, as long as you give appropriate credit to the original author(s) and the source, provide a link to the Creative Commons license, and indicate if changes were made. The images or other third party material in this article are included in the article's Creative Commons license, unless indicated otherwise in a credit line to the material. If material is not included in the article's Creative Commons license and your intended use is not permitted by statutory regulation or exceeds the permitted use, you will need to obtain permission directly from the copyright holder. To view a copy of this license, visit http://creativecommons.org/ licenses/by/4.0/

(C) The Author(s) 2017 